Methods: An electronic diary (ED) run on mobile phones was trailed with eight young people recruited from a youth health clinic that regularly drank alcohol. The ED assessed alcohol use, mood, stresses and activities. Participants were asked to complete the ED four times each day for 1 week and provide feedback about the diary's effectiveness and ease of use.

Results: Drinking alcohol was associated with cooking/eating and study/homework. While drinking, participants were mostly alone $(33.3 \%)$ or with their partner $(33.3 \%)$ and drank to relax $(33.3 \%)$ or feel good (16.7\%). Participants generally had low negative mood while drinking and drank from 0 to 5 times a week.

Conclusions: After some setbacks, the ED was successfully implemented in a clinical setting. Responses to the diary provided a rich variety of information including information on mood, stresses, activities, alcohol and marijuana use. Participants' feedback on how they found the diary was also useful and provided good suggestions on how to improve the diary for further studies.

\section{The SHADE Project: self-help for alcohol/other drug use and depression}

\author{
F Kay-Lambkin', V Carrr, ${ }^{1,2}$, Lewin' ${ }^{1}$, B Kelly ${ }^{3}$, A Baker ${ }^{1}$ \\ ${ }^{1}$ Centre for Mental Health Studies, University of Newcastle; ${ }^{2}$ NISAD; and \\ ${ }^{3}$ Centre for Rural and Remote Mental Health, New South Wales, Australia
}

Background: The co-occurrence of depression and alcohol/other drug misuse is more common than expected by chance alone. Despite this, an effective program of treatment is yet to be established for people experiencing this comorbidity. This is a concern, given rates of depression and alcohol misuse are on the increase.

Aim: This paper will report on the posttreatment alcohol/other drug- and depression-related outcomes of the SHADE project, a large-scale, multisite study of computerized psychological treatment.

Methods: SHADE participants were those with current levels of depression and current problematic use of alcohol, cannabis or amphetamines. Following an initial assessment, participants received one face-toface case formulation session with a therapist and were subsequently randomized to receive nine sessions of SHADE therapy through a therapist, nine sessions of SHADE therapy through a computer or nine sessions of person-centered (supportive) counseling. Follow-up occurred at posttreatment, 6- and 12-month follow-up.

Results: Posttreatment results will be reported for the 250 participants recruited to the study in rural/remote and urban NSW.
Conclusions: Computerized treatment is not meant as a stand-alone therapy. The results from this study suggest that computer-based interventions can produce important gains for people with depression and alcohol/other drug use comorbidity. Further implications will be discussed.

\section{Mental health first aid standards for self-harm}

\author{
C Kelly, R Langlands, A Jorm
}

ORYGEN Research Centre, Melbourne, Australia

Background: Mental disorders are highly prevalent, but many people do not seek help for them. We aim to develop mental health first aid standards for a number of mental disorders and related crises. Deliberate, nonsuicidal self-injury is becoming more common, particularly among young people. Little is known about how to prevent repetition or support someone who is deliberately harming him/herself.

Methods: The methodology of this study is the 'Delphi method', a technique for determining consensus. Initially, we searched for claims and suggestions about how to help someone who engages in deliberate selfharm. Searching the medical literature, carer's manuals, memoirs, pamphlets and Web sites, very little was found. Round 1 of this study instead asked people to write in their own words what might be the right thing to do. Questions have been generated from these written answers. Respondents are clinicians or researchers, carers and consumers from Australia, New Zealand, Canada, the UK and the United States.

Results: The majority of the participants felt that 'stopping someone' from injuring him/herself is counterproductive. The two emphases are on dealing medically with any serious injuries and allowing the person space to talk about his/her distressing feelings.

Conclusions: These standards will be used in Mental Health First Aid training and will be widely available to members of the public who are concerned about a family member or friend who is engaging in self-injury.

\section{Vocational rehabilitation in first- episode psychosis: results of the first Australian randomized controlled trial of individual placement and support}

\section{E Killackey 1,2, P McGorry ${ }^{1,3}$, K Roffel', G Chinnery', H Jackson ${ }^{2}$}

'ORYGEN Research Centre; 2Department of Psychology, The University of Melbourne; and ${ }^{3}$ ORYGEN Youth Health, Melbourne, Australia 
Background: Surveys consistently show that the top priority of people with mental illness is participation in the open labor market. Despite this finding, the employment outcome for people with psychotic illnesses is not good. At the onset of illness, unemployment rates of $40 \%-50 \%$ are commonly found. For those who develop schizophrenia, unemployment rises to $70 \%-95 \%$. These figures are troubling to consumers, clinicians and politicians. Individual Placement and Support (IPS) is a vocational intervention, which has been developed and trailed successfully in populations with chronic serious mental illness in America. To date, there has been no published randomized trial of IPS in early mental illness. This study aimed to examine the efficacy of IPS in a randomized controlled trial with people with first-episode psychosis.

Methods: Clients of EPPIC at ORYGEN in Melbourne who wished to find work were randomized to treatment as usual (TAU) $(n=20)$ or TAU + IPS $(n=20)$. The IPS condition involved working with an employment consultant who was integrated with the mental health team.

Results: Results to be presented will show that clients in the IPS group achieved greater employment outcomes than those in the TAU-only group. Other results will be presented examining symptomatic and functioning factors.

Conclusions: There is an increasing recognition that the rehabilitation of people with mental illness needs to take into account functional as well as symptomatic domains. Although there are structural obstacles making this more difficult, the current project shows what is possible with minimal extra resources.

\section{Diagnosing mild cognitive impairment - a data-driven approach}

\section{N Kochan, P Sachdev, J Trollor, H Brodaty, M Slavin}

School of Psychiatry, University of New South Wales, Sydney, Australia

Background: There has been increasing research interest in the concept of mild cognitive impairment (MCI) as a prodrome to Alzheimer's disease and other dementias. Several diagnostic schemas have been proposed with a central feature being the presence of cognitive impairment in one or more domains. But how should cognitive impairment be determined? Which tests, how many and what cut-offs should be used? The current study uses a data-driven approach to determine patterns of healthy cognitive functioning and impairment in a community sample of older adults.

Methods: Four hundred adults aged 70-90 years completed a comprehensive neuropsychological assessment as part of the Memory and Ageing Study, Sydney.
Results: Prevalence of cognitive impairment across the domains of memory, language, psychomotor speed, visuospatial and frontal-executive functions varied considerably when different sources of normative data, demographic corrections, cut-scores and clusters of tests were applied.

Conclusions: $\mathrm{MCI}$ is a very difficult construct to define at an individual and group level. This study provides much needed normative neuropsychological data in an Australian older adult sample. Longitudinal data will inform us about the most sensitive and specific neuropsychological profile that will predict those who progress to dementia.

\section{The effects of adjunctive estradiol on cognitive performance in women with schizophrenia}

\section{J Kulkarni, C Bartholomeusz, C Gurvich, S Sheppard, $S$ White, A de Castella, P Fitzgerald, P Nathan}

Alfred Psychiatry Research Centre, Melbourne, Australia

Background: Development of pharmacological strategies for improving cognitive impairment has recently become a key issue in the treatment of schizophrenia. The steroid hormone estrogen is hypothesized to be protective for women with schizophrenia and has been found to exert positive effects on specific cognitive domains in healthy postmenopausal women. We have previously reported a significant improvement in psychopathology ratings associated with adjunctive estrogen treatment. We additionally investigated the effects of adjunctive estrogen treatment on cognitive function in women with schizophrenia.

Methods: Fifty women of childbearing age with schizophrenia or schizoaffective disorder received $100 \mu \mathrm{g} /$ day transdermal estradiol or placebo for 4 weeks, under double-blind conditions. The cognitive battery, assessing attention, verbal fluency, memory and executive function, was administered at baseline and at 4 weeks. Hormone assays were collected, and psychopathology was measured weekly.

Results: Results indicated no significant changes in cognition following 4 weeks of adjunctive estrogen treatment. While baseline endogenous estrogen levels were also not significantly related to cognitive function, there was a correlation found between $\mathrm{LH}$ and a measure of information processing.

Conclusions: Short-term estrogen treatment as an adjunct to antipsychotics does not significantly alter $\operatorname{cog}$ nitive functioning, despite significant improvements in psychopathology ratings. It may be that estrogen treatment has selective effects on psychopathology; 